\title{
Optimización Simultánea del Diseño Conceptual de Procesos y Layout de Planta Considerando el Riesgo Tecnológico
}

\section{Simultaneous Optimization of Conceptual Process Design and Plant Layout Considering Technological Risk}

Presentación: 06/10/2020

\section{Doctorando:}

\section{Santiago Orellano}

Centro de Aplicaciones Informáticas y Modelado en Ingeniería (CAIMI), Facultad Regional Rosario (FRRo), Universidad Tecnológica Nacional (UTN)

santiago.orellano93@gmail.com

\section{Director:}

\section{Nicolás José Scenna}

\section{Co-director:}

\section{Néstor Hugo Rodríguez}

\section{Resumen}

En este trabajo se presentan brevemente los avances y resultados desarrollados en el marco de la tesis doctoral titulada "Metodología para la Optimización de Layout y Síntesis de Procesos Considerando el Riesgo Tecnológico". A través de la misma se pretende desarrollar un algoritmo eficiente que permita la obtención de la distribución óptima de las unidades de proceso y de los departamentos presentes en el complejo industrial bajo la consideración de la filosofía de diseño inherentemente seguro, incluyendo las principales restricciones de diseño estructural. Para la implementación de tal algoritmo, se han desarrollado modelos de optimización que permiten vincular la toma de decisiones en la etapa del diseño conceptual y del diseño del layout a través de la contemplación de las aristas comunes entre ambas etapas, considerando tanto la vulnerabilidad del interior de la planta como la del entorno (viviendas, escuelas, hospitales, etc.). Esta vinculación se llevó a cabo mediante el desarrollo de modelos reducidos para la evaluación del impacto ante distintos eventos accidentales.

Palabras clave: Optimización de Layout, Diseño Basado en Riesgo, Playa de tanques, Incendio de Charco.

\begin{abstract}
This paper briefly presents the progress and results developed within the framework of the PhD thesis entitled "Methodology for Layout Optimization and Process Synthesis Considering Technological Risk". Through it, it is intended to develop an efficient algorithm that allow to obtain the optimal distribution of the process units and the departments present in the industrial plant under the consideration of the inherently safe design philosophy, including the main structural design restrictions. For the implementation of such an algorithm, optimization models have been developed that allow to connect decision-making in the conceptual design stage and in the layout design stage through the contemplation of the edges common to both stages, considering both the vulnerability of the interior of the plant and the surroundings (homes, schools, hospitals, etc.). This link was carried out through the development of reduced models for the evaluation of the impact of different accidental events
\end{abstract}

Keywords: Layout Optimization, risk-based design, Tank Farm, Pool Fire. 


\section{Introducción}

En las últimas tres décadas, se ha impuesto la sostenibilidad de los procesos; lo cual, entre otros factores, implica seguridad operativa y de diseño de los mismos. Han sido difundidas ampliamente dos estrategias de diseño: la filosofía de diseño inherentemente seguro y la estrategia de diseño basado en riesgo.

En paralelo a esto, se publicaron diversos artículos para resolver el problema de optimización de layout a través de programación matemática y de la aplicación de técnicas de optimización. Pueden citarse dos corrientes bien diferenciadas: por un lado, se utilizaron métodos determinísticos de optimización que, para lograr un cierto grado de rigurosidad, resultan limitados en la cantidad de unidades posibles de tratar (Jung et al., 2011; Medina-Herrera et al., 2014; entre otros) y por otro lado, se aplicaron técnicas de optimización meta-heurísticas (Genetics algorithm, Bat Methaheuristics algorithm, Tabu Search, Simulated annealing, entre otras) (Caputo, 2015; Latifi et al., 2017; entre otros), con la limitación de no poder garantizar los atributos de la solución óptima obtenida.

Estos modelos permitieron evitar el diseño iterativo del layout mediante la evaluación posterior del riesgo. A pesar de la gran ventaja que supone esto, las metodologías determinísticas actuales adolecen de diversas carencias si se pretende resolver problemas de optimización de layout realistas considerando el cálculo del riesgo. Dos de los motivos limitantes son el crecimiento combinatorio exponencial en función de las unidades de procesamiento (UP) a contemplar; y la complejidad asociada a la inclusión de la función Probit para el cálculo de la vulnerabilidad del entorno (y, por lo tanto, del riesgo).

Considerando la limitación de los métodos determinísticos, se desarrollaron diversas metodologías para la resolución de los problemas de layout con un elevado número de unidades. En base a los trabajos existentes en la literatura, hemos propuesto (en base a la metodología desarrollada por Xu y Papageorgiou (2009)), una nueva metodología basada en la resolución secuencial de la optimización del layout considerando la estrategia de diseño inherentemente seguro (Orellano et al., 2020). Sintéticamente, consiste en definir un conjunto de $m$ unidades del total que se localizarán mediante un modelo de optimización mixto entero lineal (MILP). Una vez determinada las coordenadas y orientaciones de éstas, se agregan secuencialmente conjuntos de $n$ unidades mediante la fijación de las variables ya determinadas previamente. El algoritmo se repite hasta ubicar la totalidad de las unidades. Es necesario resaltar que, a pesar de que esta metodología no garantiza la globalidad de la solución óptima obtenida, permite la obtención de soluciones eficaces.

En particular, se pretende vincular las variables de diseño con importante repercusión en el riesgo (las cuales son fácilmente identificables a partir de un análisis preliminar del riesgo -PHA-) con la optimización del layout. Se han implementado modelos de optimización que permiten determinar simultáneamente la localización de estas unidades y el diseño de las mismas. Es necesario resaltar que para poder vincular una variable de diseño con el problema de layout es necesario contar con modelos simplificados/reducidos para la determinación de distancias mínimas aceptables (de modo de garantizar un nivel de impacto máximo sobre los receptores dado un potencial evento accidental). Estos modelos deben ser lo suficientemente sencillos para poder incorporarlos en un modelo de optimización y a la vez, lo suficientemente precisos (mínima sobreestimación del impacto/distancia), ya que de ello depende la bondad de la solución óptima obtenida. Considerando esto, se ha desarrollado un modelo para la estimación de la distancia/impacto dado el evento pool fire (aplicable a trench fire dadas ciertas condiciones).

\section{Desarrollo}

Los problemas derivados del FLP (Facility Layout Problem) pertenecen a los NP-Hard Problem aún en las formulaciones más simples del mismo. En función de esto, es ilógico pretender resolver grandes problemas de layout garantizando la globalidad de la solución óptima obtenida, y menos aún si se busca, además de optimizar el layout dados ciertos distanciamientos mínimos, correlacionar estos distanciamientos en función de variables de diseño y de las características de los receptores. A partir de ello, se desarrollaron gradualmente, en una escala de complejidad creciente, distintos ejemplos contemplando diversos números de unidades a localizar y la etapa de diseño en que se encuentran; con el objetivo de desarrollar una metodología que permita la obtención de soluciones aceptables.

La optimización del layout considerando riesgos puede darse en distintas circunstancias y etapas del diseño, por lo que la metodología para la obtención de la "solución óptima" variará en función de ello. Consideremos el diseño completo del layout: 
si el entorno en el que se desarrollará el complejo industrial no incluye potenciales receptores ante un evento accidental, se podrá desarrollar un determinado criterio jerárquico para la optimización (a los efectos de particionar el problema global en etapas); mientras que, si existieran puntos de interés externos, este criterio diferirá. Por otro lado, si la optimización se realiza sobre un complejo industrial ya instalado y deben localizarse nuevas unidades (cambio en la materia prima, un caso de revamping, entre otros), sería posible, bajo ciertas condiciones, la obtención de óptimos globales incluso considerando la determinación de variables de diseño conceptual. Este trabajo pretende plantear la problemática, resumir el estado del arte, los avances desarrollados y bosquejar una primera aproximación para la generalización de la metodología propuesta.

A continuación, y en forma sintética, se presenta la estructura lógica del algoritmo jerárquico propuesto (aún en desarrollo) para el diseño óptimo del layout de planta considerando el diseño inherentemente seguro.

\section{Etapa 0: Pre-procesamiento}

En la etapa de pre-procesamiento es necesario identificar principalmente la siguiente información:

- Caracterizar la etapa de desarrollo del layout: La metodología a seguir dependerá de si es necesario definir el layout del complejo industrial, planta de proceso, almacenamientos, entre otros; o bien, el objetivo consiste en una ampliación de la planta o un caso de revamping.

- Identificar las características del entorno: Será necesario definir, independientemente de la etapa de desarrollo del layout en que se encuentre, la descripción del entorno del complejo industrial. Esto es, definir si existen puntos de interés externos (vecindarios, otras plantas, etc.), y en caso de existir, sus coordenadas y dimensiones en función del sistema de referencia que se adopte.

\section{Etapa 1: Identificación de bloques o componentes del complejo objeto de estudio}

Esta primera etapa consiste en agrupar las unidades de proceso (UP) en bloques (a partir de una serie de criterios) con el objetivo de independizar la optimización rigurosa del layout interno de dichos bloques y la del layout general de planta (blockout). Esto permite reducir el número de modelos que se resuelven en simultáneo, esto es, realizar una partición del problema, introduciendo la necesidad de definir jerarquías en el orden de resolución de los respectivos problemas en un orden secuencial.

En cuanto a los criterios para agrupar UP en los distintos bloques, se consideran aquí las técnicas heurísticas sugeridas para realizar el agrupamiento de las unidades (CCPS, 2003 y Mannan S., 2004), ya que a partir de estas se reduce notablemente la probabilidad del efecto dominó. Por ejemplo, la incompatibilidad del almacenamiento de sustancias (CCPS, 1995), la localización de las unidades operando a presión atmosféricas con cierto distanciamiento de las unidades a presión, lo cual contribuye notablemente en la reducción de la probabilidad de efecto dominó (de Lira Flores et al., 2018).

Los grupos/o bloques usuales en que se pueden agrupar las diversas UP son:

- Bloque de Proceso (i)

- Bloque de Almacenamiento $(j)$

- Bloque de Personal ( $k$ )

- Bloque de Servicios auxiliares (m)

A partir de este agrupamiento de UP en los distintos bloques, se debe definir la secuencia de problemas o modelos de optimización según la etapa jerárquica de resolución que corresponda.

\section{Etapa 2: Identificación de variables de diseño críticas y modelo de optimización en el que intervienen}

En esta etapa, se pretende identificar mediante un Análisis Preliminar de Riesgos (PHA) los puntos sensibles y variables relevantes en cada bloque (o sub-bloque). Es decir, se busca identificar aquellas variables de diseño que tienen el potencial de influir en la posterior optimización del layout. Por ejemplo, la determinación del número de tanques en que se almacena una sustancia peligrosa, la relación de esbeltez de estos, su layout interno, la presencia de tabiques en el endicamiento, entre otros; las cuales adquieren vital importancia a la hora del diseño del layout general. La vinculación surge, en la mayoría de los casos, mediante un trade off entre la decisión que implica menor costo y la que implica menor riesgo. En una playa de almacenamiento 
en que se desea almacenar un volumen fijo de determinada sustancia, desde una perspectiva económica, es siempre apetecible minimizar el número de tanques en que este volumen se dispone. Sin embargo, desde el punto de vista del riesgo, a medida que se aumenta el número de tanques, el impacto ante un potencial evento accidental disminuye, ya que la masa involucrada en el accidente sería menor. Luego, aquí se observa claramente el trade off si se pretende realizar un diseño inherentemente seguro: aumentar la inversión en la etapa de decisión correspondiente al diseño del bloque de almacenamiento, podría traer aparejado una reducción de los costos en el layout, ya que se reduciría la distancia aceptable hacia las demás unidades y, por lo tanto, los costos del terreno y de la conectividad, entre otros.

Además de definir las variables de diseño que ingresan en el modelo de optimización de layout, es necesario identificar en qué orden jerárquico se define su optimización. Es decir, si la definición de esta variable impacta mayormente en el layout del bloque en sí mismo, o bien, a nivel de la definición del blockout. Esto obviamente tiene implicancias en el planteo de cada problema de optimización y la secuencia jerárquica de resolución de los mismos.

Como mencionamos previamente, es indispensable contar con modelos reducidos para la determinación de distancias mínimas aceptables. Se desarrolló un modelo reducido para el cálculo de la distancia de impacto dado un eventual incendio de charco (pool fire). Para la obtención del mismo, se realizó una búsqueda y reproducción exhaustiva de los modelos rigurosos desarrollados para el cálculo del impacto. En función de los resultados arrojados por estos, se desarrollaron modelos para el cálculo del impacto (o de la distancia segura) a través de funciones simples.

Sean, RM: Modelo de Referencia e IRM: Modelo Reducido Inverso. A partir del modelo base (RM), se obtuvo un modelo semejante, aunque más sencillo, que llamamos $(D R M)$, y otro, también más sencillo que el $R M$, pero además "inverso", en el sentido en que la variable de salida es la distancia segura de impacto (IRM).

- HRR (Propiedad de la sustancia o mezcla)

- I (Impacto determinado sobre el receptor)

- D (Diámetro equivalente del incendio)
IRM

L (Distancia desde la pared del incendio)

La función obtenida es del tipo $L=f(H R R, I, D)$, donde $H R R$ representa el calor de combustión por unidad de área (Heat Release Rate), que es un parámetro característico de la sustancia; $I$, el impacto máximo preestablecido sobre los distintos receptores; y $D$, el diámetro equivalente del endicamiento. A partir de esto, considerando las normas de diseño tanto de los tanques como del endicamiento, es posible vincular el conjunto de variables de diseño conceptual que definen a la (UP) playa de almacenamiento con las dimensiones de la misma y la distancia mínima aceptable hacia las demás unidades del layout.

\section{Etapa 3: Orden jerárquico}

Una vez definidas las variables de diseño que se determinarán en simultáneo con el layout, la resolución requerirá resolver en primer lugar la estructura (layout interno) de todos los bloques en que se han agrupado las UP del complejo. En líneas generales, se mencionan a continuación las estrategias adoptadas en función de los distintos bloques:

\section{- Bloque de Proceso (i)}

La combinación de las estrategias propuestas por (Xu y Papageorgiou, 2009) y por (Mannan, 2004), resulta conveniente para el desarrollo de un eficiente layout del bloque proceso. Esto es: agrupar los equipos según la operación unitaria (o conjunto de operaciones unitarias) a la que pertenezcan (Mannan, 2004). Por ejemplo, en el caso de una torre de destilación, el agrupamiento para la definición del layout incluiría la columna en sí misma, hervidor, condensador, bombas y cañerías. Una vez realizado este agrupamiento, si el número excede la cantidad de unidades (UP) posibles de "manejar" por un algoritmo de optimización de layout determinístico, será necesaria la aplicación de la metodología propuesta por (Xu y Papageorgiou, 2009). La secuenciación de esta metodología dependerá del tipo de proceso: si el proceso es suficientemente lineal, la secuenciación siguiendo el flujo será eficiente; mientras que, si el proceso presenta un alto grado de interconectividad, la secuenciación mediante el algoritmo 
desarrollado en (Orellano et al., 2020) será más adecuada. La experiencia obtenida, y los reportes en la literatura, indican que, en el último tipo mencionado, el número de UP a optimizar sin adoptar estrategias de particionado oscila entre 10 y 15 unidades.

\section{- Bloque de Almacenamiento (j)}

El caso de las playas de almacenamiento requiere particular atención dado que la masa involucrada puede desencadenar potenciales eventos accidentales que afecten no sólo el interior de la planta, sino también el entorno de la misma. En función de la experiencia adquirida en el desarrollo de modelos de optimización de layout y diseño de playas de almacenamiento, la optimización en simultáneo de su diseño en conjunto con el blockout parece ser la alternativa más viable. Se han desarrollado modelos que permiten la optimización de su diseño y localización para casos de revamping o ampliación de planta. Para el caso particular de diseño de la planta desde cero, se han desarrollado modelos para la optimización de la playa de tanques considerando una sustancia y un evento accidental (Orellano, 2019). Dado el reciente desarrollo de diversos modelos reducidos (aún no publicados), resultará factible la optimización de sistemas de almacenamiento de diversas sustancias y contemplando distintos escenarios accidentales.

\section{- Bloque de Personal (k)}

Dado que se pretende la localización de los bloques con frecuente presencia de personas (oficinas, talleres, laboratorios, etc.) en posiciones alejadas de los sitios con cierto riesgo asociado, es conveniente incluir estos bloques en las últimas etapas de la secuenciación del blockout.

\section{- Bloque de Servicios Auxiliares (m)}

En dichos bloques se incluyen aquellos correspondientes a los servicios auxiliares del proceso: generación de vapor para calentamiento o bien para la producción de energía eléctrica o para impulsar otras UP, plantas de tratamiento de efluentes, tratamiento de agua para calderas, torres y circuitos de agua de enfriamiento, entre otros. Particularmente en aquellos bloques con cierto riesgo asociado (sala de calderas, por ejemplo) probablemente será determinante la inclusión en los modelos de layout ciertas variables de diseño de los mismos. La determinación de estos, será función del proceso a analizar. Por ejemplo, en la optimización del layout de una planta de cogeneración, la caldera formará parte del proceso en sí mismo.

\section{Etapa 4: Resolución del layout-blockout.}

La lógica para la determinación del orden de incorporación ha sido probada usando el modelo desarrollado en (Orellano, 2020), donde sólo se consideran los costos de la conectividad. Futuros trabajos se destinarán al análisis de este ordenamiento no sólo en términos económicos, sino también desde la perspectiva del riesgo. De existir variables de diseño cuya determinación se realizan en simultáneo, será conveniente la incorporación de las mismas en las primeras etapas jerárquicas (primeros conjuntos de bloques) a optimizar, puesto que la región factible para estas será más relajada que para su incorporación en etapas posteriores, con el consecuente beneficio económico.

Por otro lado, la incorporación de las unidades más "riesgosas" en las primeras etapas permite la incorporación al modelo de optimización de distancias de seguridad variables en etapas posteriores considerando variables estocásticas características del lugar donde se instala la planta (sentido y velocidad del viento) que influyen en distintos eventos accidentales como la dispersión de tóxicos o la explosión de una nube de vapor no confinada. Una vez definido esto, el problema se reduce a un problema similar al resuelto por (Medina-Herrera et al., 2014).

\section{Etapa 5: Verificación}

Una vez finalizado el layout, el método tradicional o estado del arte del diseño consiste en la examinación crítica. Esto es, responder a las siguientes preguntas para cada unidad: ¿Dónde está localizada la unidad? ¿En qué otro lugar podría colocarse? (Mannan, 2004). Las respuestas a estas preguntas pueden ser fácilmente implementadas en el algoritmo de optimización, no sólo por unidad sino también por grupos. Esta implementación consiste en dejar fijas las posiciones y orientaciones de todas las unidades a excepción de las que se evalúan mediante este criterio, lo cual implica un tiempo de cómputo pequeño si el número de 
unidades liberadas es pequeño. Incluso es factible en este punto, no sólo liberar estas variables, sino también las variables de diseño, resolviéndose así un problema análogo a un caso de ampliación de planta.

\section{Conclusiones}

A través de este trabajo, se ha desarrollado el esquema general de la metodología algorítmica en desarrollo para la optimización simultánea del layout de planta y de ciertas variables de diseño con significativo impacto en el layout, el cual representa el objetivo central de esta tesis. Se han incluido citas de los trabajos ya publicados y menciones a los trabajos que aún están en proceso de desarrollo, que forman parte de las distintas etapas mencionadas previamente.

Si bien la metodología en su totalidad no ha sido implementada aún, el desarrollo de las distintas etapas indicaría que es factible la obtención de soluciones prometedoras.

El desarrollo de tal metodología junto con los modelos de optimización que se requieren para su ejecución traerá aparejado la posibilidad de sistematizar el diseño del layout y el desarrollo de plantas más seguras.

\section{Referencias}

Caputo, A.C., Pelagagge, P.M., Palumbo, M., Salini, P., (2015). Safety-based process plant layout using genetic algorithm. Journal of Loss Prevention in the Process Industries, 34: 139-150.

Center for Chemical Process Safety, (1995). Guidelines for Safe Storage and Handling of Reactive Materials. New York, Wiley.

Center for Chemical Process Safety, (2003). Guidelines for siting and layout of facilities (2nd ed.). New York, Wiley.

de Lira-Flores J.A., Gutiérrez-Antonio C., Vázquez-Román R. (2018). A MILP approach for Optimal Storage Vessels Layout based on the Quantitative Risk Analysis Methodology, Process Safety and Environmental Protection. 120: 1-13.

Jung, S. Facility sitting and layout optimization based on process safety. Thesis for the degree of Doctor of Philosophy, Texas A\&M University. (2010).

Latifi, S., Mohammadi, E., Khakzad N. (2017). Process plant layout optimization with uncertainty and considering risk. Computers and Chemical Engineering, 106: 224-242

Mannan, S. (2004). "Plant Siting and Layout". Lees' Loss Prevention in the Process Industries. Hazard Identification, Assessment and Control (3rd ed.) Cap 10. Texas, Elsevier Inc.

Medina-Herrera, N., Jiménez, G., Grossman, I.E. (2014). A mathematical programming model for optimal layout considering quantitative risk analysis. Computers and Chemical Engineering, 68: 165-181.

Orellano, S.; Rodríguez, N.; Scenna, N. J. (2019). Optimización de playas de almacenamiento de sustancias peligrosas frente a escenarios de trench fire. CAIQ2019. Santa Fe.

Orellano, S., Rodríguez, N., \& Scenna, N. (2020). Metodología Algorítmica para el Diseño Inherentemente Seguro de Planta. Revista Tecnología Y Ciencia, (38), 1-17. https://doi.org/10.33414/rtyc.38.1-17.2020

Xu G.; Papageorgiou L. G. (2009). Process plant layout using an improvement-type algorithm. Chemical Engineering Research and Design. 87: 780-788. 